\title{
UCRL-CONF-223806
}

LAWRENCE LIVERMORE N A T IO N A L LABORATORY

\section{Spectral Line Shapes as a Diagnostic Tool in Magnetic Fusion}

R. Stamm, H. Capes, A. Demura, L. Godbert-Mouret, M. Koubiti, Y. Marandet, M. Mattioli, J. Rosato, F. Rosmej, K. B. Fournier

August 18, 2006

18th International Conference on Spectral Line Shapes Auburn, AL, United States June 1, 2006 through June 30, 2006 
This document was prepared as an account of work sponsored by an agency of the United States Government. Neither the United States Government nor the University of California nor any of their employees, makes any warranty, express or implied, or assumes any legal liability or responsibility for the accuracy, completeness, or usefulness of any information, apparatus, product, or process disclosed, or represents that its use would not infringe privately owned rights. Reference herein to any specific commercial product, process, or service by trade name, trademark, manufacturer, or otherwise, does not necessarily constitute or imply its endorsement, recommendation, or favoring by the United States Government or the University of California. The views and opinions of authors expressed herein do not necessarily state or reflect those of the United States Government or the University of California, and shall not be used for advertising or product endorsement purposes. 


\title{
Spectral Line Shapes as a Diagnostic Tool in Magnetic Fusion
}

\author{
R. Stamm ${ }^{1}$, H. Capes ${ }^{2}$, A. Demura ${ }^{3}$, L. Godbert-Mouret ${ }^{1}$, M. Koubiti ${ }^{1}$ \\ Y. Marandet ${ }^{1}$, M. Mattioli ${ }^{4}$, J. Rosato $^{1}$, F. Rosmej $^{1}$ and K. B. Fournier ${ }^{5}$ \\ ${ }^{1}$ Physique des Interactions Ioniques et Moléculaires \\ Université de Provence, Marseille, France \\ ${ }^{2}$ Département de Recherches sur la Fusion Contrôlée, \\ CEA, Cadarache, France \\ ${ }^{3}$ HEPTI, RRC "Kurchatov Institute", Moscow, Russia \\ ${ }^{4}$ Consorzio RFX, Associazzone Euratom-Enea sulla Fusione, Padova, Italy \\ ${ }^{5}$ Lawrence Livermore National Laboratory, P.O. Box 804, Livermore, CA 94551, USA
}

\begin{abstract}
Spectral line shapes and intensities are used for obtaining information on the various regions of magnetic fusion devices. Emission from low principal quantum numbers of hydrogen isotopes is analyzed for understanding the complex recycling mechanism. Lines emitted from high principal quantum numbers of hydrogen and helium are dominated by Stark effect, allowing an electronic density diagnostic in the divertor. Intensities of lines emitted by impurities are fitted for a better knowledge of ion transport in the confined plasma.
\end{abstract}

Keywords: Zeeman effect, Stark broadening, Doppler broadening, edge plasmas, turbulence, transport

PACS: 32.70.Jz, 52.25.Gj, 52.25.Os, 52.55.Fa, 52.70.-m

\section{INTRODUCTION}

Similarly to spectral observations and modelings in astrophysics, researches in line broadening for magnetic fusion devices are inspired by a vision. One dreams that the line shapes will easily convey all the information on the plasma containing the emitter, revealing density and temperature of the charged and neutral particles, the bulk motions of the plasma, and the small scale turbulent fluctuations. Such hope is however only partially fulfilled, since an accurate determination of the plasma parameters generally requires a careful and complex analysis, combining detailed data in atomic physics, a knowledge of the plasma statistical properties, and in some cases a description of hydrodynamic turbulence.

Spectral line widths and shifts have been widely used for determining the plasma parameters and dynamics since the early days of research in magnetic fusion [1]. While these first observations were made in the UV and XUV range, and concerned impurities in the core plasmas, more recent studies were mainly devoted to the emission of hydrogen isotopes in the edge plasma. These studies enabled a better understanding of the edge plasma composition and behavior, and helped to elucidate the complex recycling processes of the hydrogen isotopes [2]. Part of the studies were 
focused on the measure of the electron density by using the Stark broadening of high principal quantum number transitions of the Balmer series of deuterium [3]. Other informations like the normal and reversed ion flows in the DIII-D divertor [4] have been obtained thanks to line shapes observations of carbon ions.

In this paper, we will review some recent analyses of spectral line shapes and intensities observed in tokamaks like TORE-SUPRA, TEXTOR, JET or ALCATOR C-MOD. We will show that, depending on the plasma parameters and on the line observed, Doppler, Stark broadening and Zeeman effect may be observed, sometimes superimposed. Our toolbox for modeling such spectra is composed of population kinetic models, line shape codes, including ultra-fast Lyman line profile calculations designed for a study of line radiation transport. In a first section, we will discuss the problem of neutrals in the edge and divertor plasmas, and illustrate with the examples of hydrogen isotopes and neutral helium the dominant broadening mechanisms in the various plasmas encountered. The specific problem of line shapes for opacity calculation will be discussed for the Lyman alpha line. For divertor plasmas, and for high density plasmas such as expected in ITER, Stark broadening will be discussed in the second section. The third section will be devoted to the emission of impurity lines, generally observed in the confined core plasma. A fit of the measured spectral lines usually requires the use of accurate atomic data and an impurity transport model. Several examples will be presented, with measured data obtained on the JET tokamak. Finally, we will discuss the effect on the line shapes of the ubiquitous turbulent fluctuations of the hydrodynamic variables [5], and briefly present a statistical formalism able to take their effect into account on the observed line shapes.

\section{NEUTRAL EMISSION IN EDGE AND DIVERTOR PLASMAS}

\section{Hydrogen Isotopes in an Ionizing Plasma}

Line emission of hydrogen isotopes is a unique source of information for understanding the composition and dynamics of the edge plasma. On all tokamaks, one observes a strong emission of the optically thin Balmer alpha line. For most presently operating tokamaks, this line is broadened by Doppler effect and exhibits a structure sensitive to the magnetic field. A multi Gaussian fit of this line revealed the presence of at least three populations having different temperatures, with the coldest one being as low as $0.2 \mathrm{eV}$ [6]. This cold population actually gives the sharpest structure of such lines, which in a first approximation appear as being separated in a Lorentz triplet (or doublet in an observation parallel to the magnetic field), broadened by Doppler effect in the conditions of most current tokamaks. It has soon been realized that these different populations of neutral deuterium are generated by the various recycling mechanisms [2], each one leaving a specific signature on the $D_{\alpha}$ line shape. In all cases, a cold population with a temperature of the order of the $\mathrm{eV}$ or less results from the dissociation of deuterium molecules released by the wall. Warmer populations with a temperature of about $10 \mathrm{eV}$ or more are produced by the reflection of a neutral following the impact on the wall of an incident ion, or by charge exchange with an energetic ion. Some care should be taken for the modeling 
of this line shape, first for the case of the charge exchange population, for which a neutral transport calculation should be carried out [7]. Moreover, for the central part of the line resulting mainly from the cold atom population, a detailed structure of the line retaining fine structure and Zeeman effect should be used, especially for low magnitude magnetic field cases. Indeed, a calculation of $\mathrm{D}_{\alpha}$ for a magnetic field of 1 $\mathrm{T}$, a density $\mathrm{N}_{\mathrm{e}}=10^{12} \mathrm{~cm}^{-3}$ and an electronic temperature $\mathrm{T}_{\mathrm{e}}=1 \mathrm{eV}$, reveals that the complex structure of the line has to be taken into account for an accurate diagnostic.

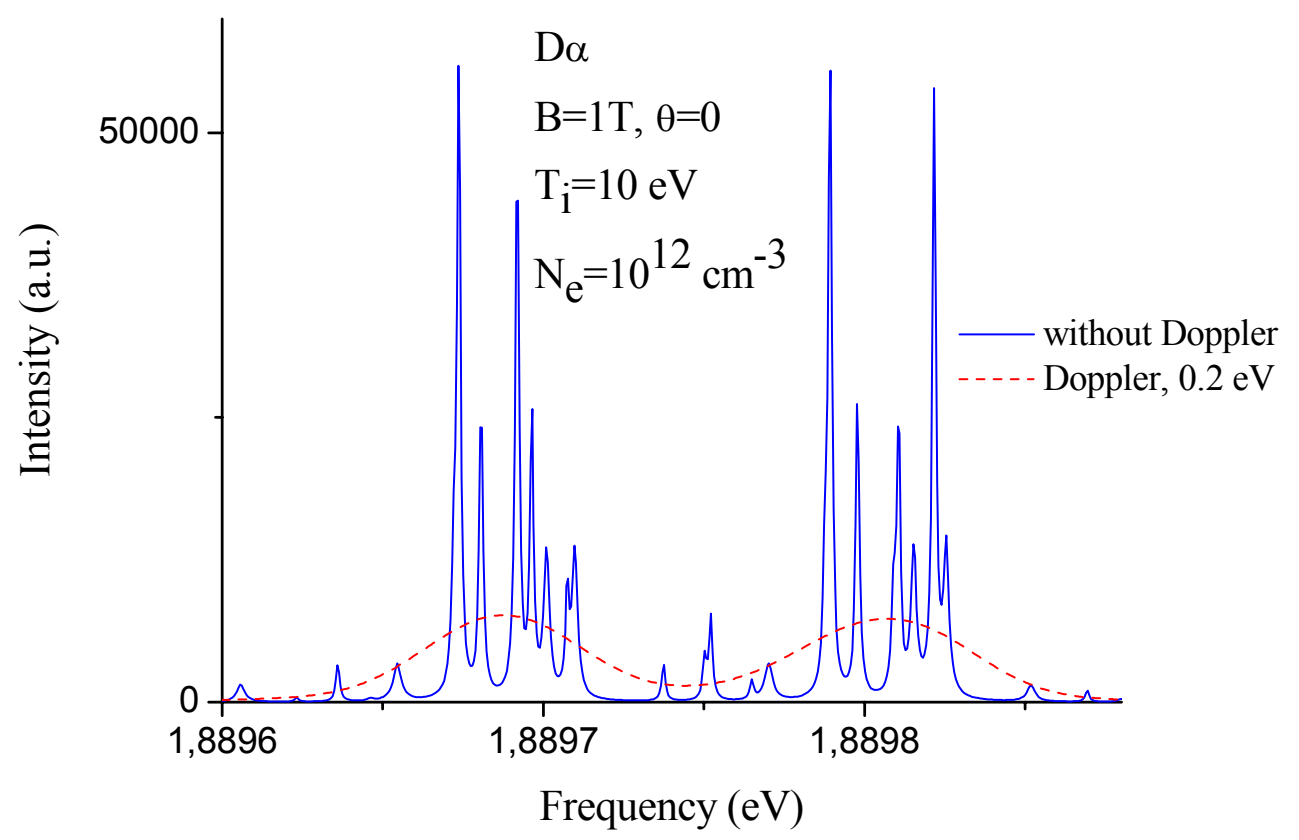

FIGURE 1. Profile of the deuterium Balmer alpha line for edge plasma conditions and an observation parallel to the magnetic field. Zeeman, Stark and fine structure effects are retained.

On Fig. 1, the numerous resulting components are drawn with a full line for the case without Doppler effect, with each component broadened by Stark effect treated in the impact approximation both for electrons and ions. If a convolution with a Gaussian Doppler profile at $0.2 \mathrm{eV}$ is performed (dotted line), it can be seen that most of the structure is lost, but a closer look reveals that the resulting profile remains asymmetric.

\section{Line Shapes for Opacity Calculations}

For the conditions of the ITER divertor, the mean free path of a Lyman alpha photon may be shorter than $2 \mathrm{~mm}$, and the plasma is thus optically thick for this radiation. Models show that this can strongly modify the ionization-recombination balance. Recently, a photon transport study was developed for the Monte-Carlo neutral transport code EIRENE [8,9], and showed the need for incorporating detailed line shapes in the modeling. This has been the motivation for providing an ultra fast, but accurate line shape model for the optically thick lines. For the Lyman alpha line, and 
for most of the plasma conditions encountered in edge plasmas, the main broadening mechanisms are Doppler, Zeeman and Stark effect. Since the final convolution with the simulated velocity distribution function is performed by the transport code, we present on Fig. 2 the Lyman alpha line shape without Doppler effect, but retaining Zeeman and Stark effect. Here the calculation has been performed analytically, by using an impact approximation for both ions and electrons $[10,11]$.

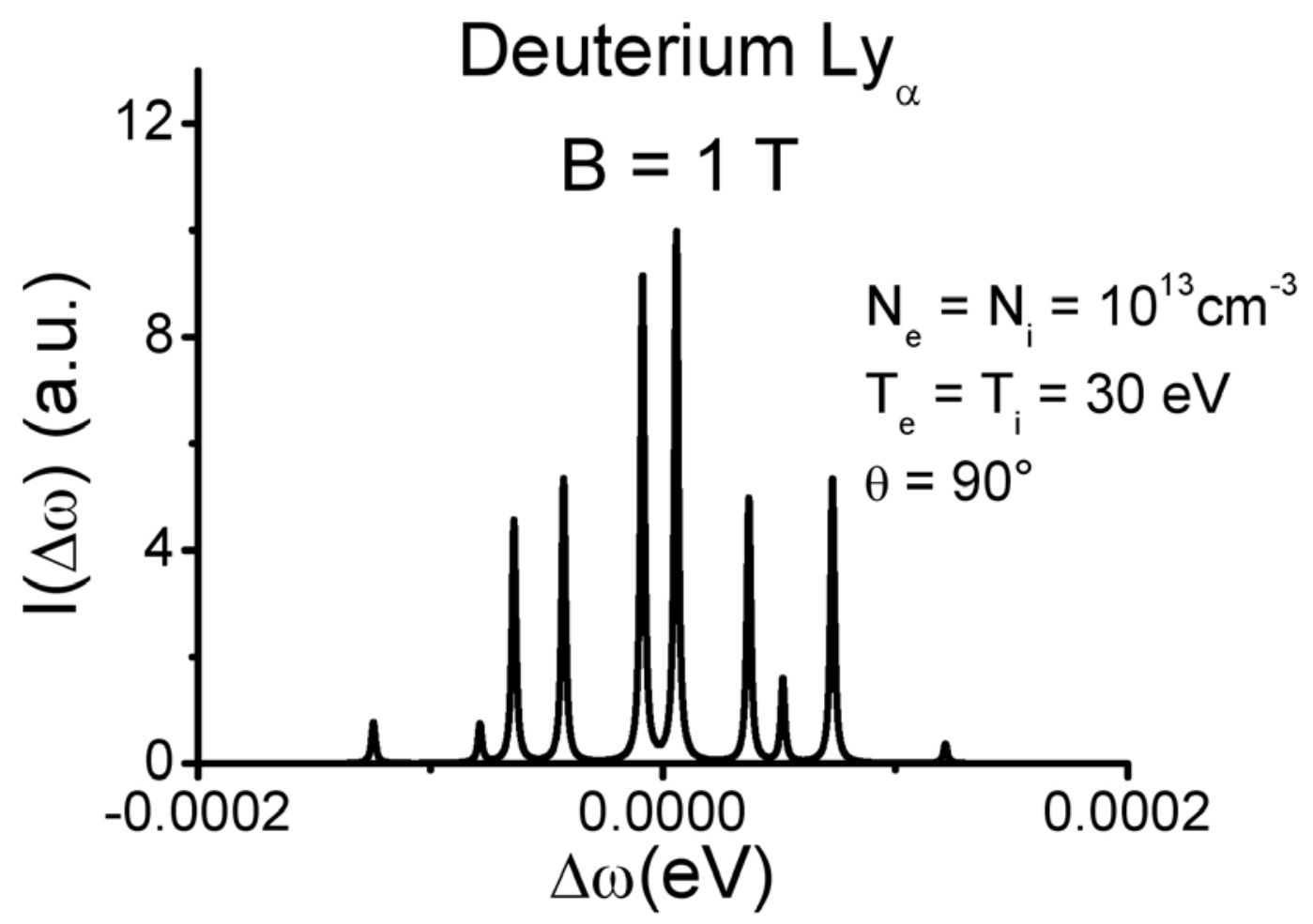

FIGURE 2. Profile of Lyman alpha line for edge plasma conditions and an observation perpendicular to the magnetic field $\left(\theta=90^{\circ}\right)$. Zeeman, Stark and fine structure effects are retained.

Using this modeling, it can be seen on Fig. 2 that Lyman alpha consists in ten components for this observation perpendicular to the magnetic field, and that the asymmetry resulting from the fine structure of the line is clearly seen. For the conditions of this calculation, with $\mathrm{N}_{\mathrm{e}}=10^{13} \mathrm{~cm}^{-3}$ and equal electronic and ionic temperature, $\mathrm{T}_{\mathrm{e}}=\mathrm{T}_{\mathrm{i}}=30 \mathrm{eV}$, the validity of the impact approximation for the ions is well insured. Using the EIRENE transport code, calculations of the ionization balance for the conditions of the ITER divertor are in progress by using these detailed profiles. For higher densities and/or lower temperatures, the Stark broadening model for ions should take into account the effect of many body dynamic interactions with the emitter. These conditions will be discussed in the next section. 


\section{ROLE OF STARK EFFECT}

\section{Recombining Divertor Plasmas}

In the conditions of most presently operating tokamaks, Stark effect mainly affects hydrogen isotopes or helium lines originating from high principal quantum numbers. Such lines are often seen in divertor plasma conditions, where three body recombinations are followed by radiative cascades allowing the observation of transitions with upper principal quantum number as high as $n=15-20$. On Fig. 3, we have plotted a part of the spectra of He I $1 \mathrm{~s} 2 \mathrm{p}{ }^{3} \mathrm{P}-1 \mathrm{snd}{ }^{3} \mathrm{D}$ diffuse line series, observed in the divertor of the JET tokamak.

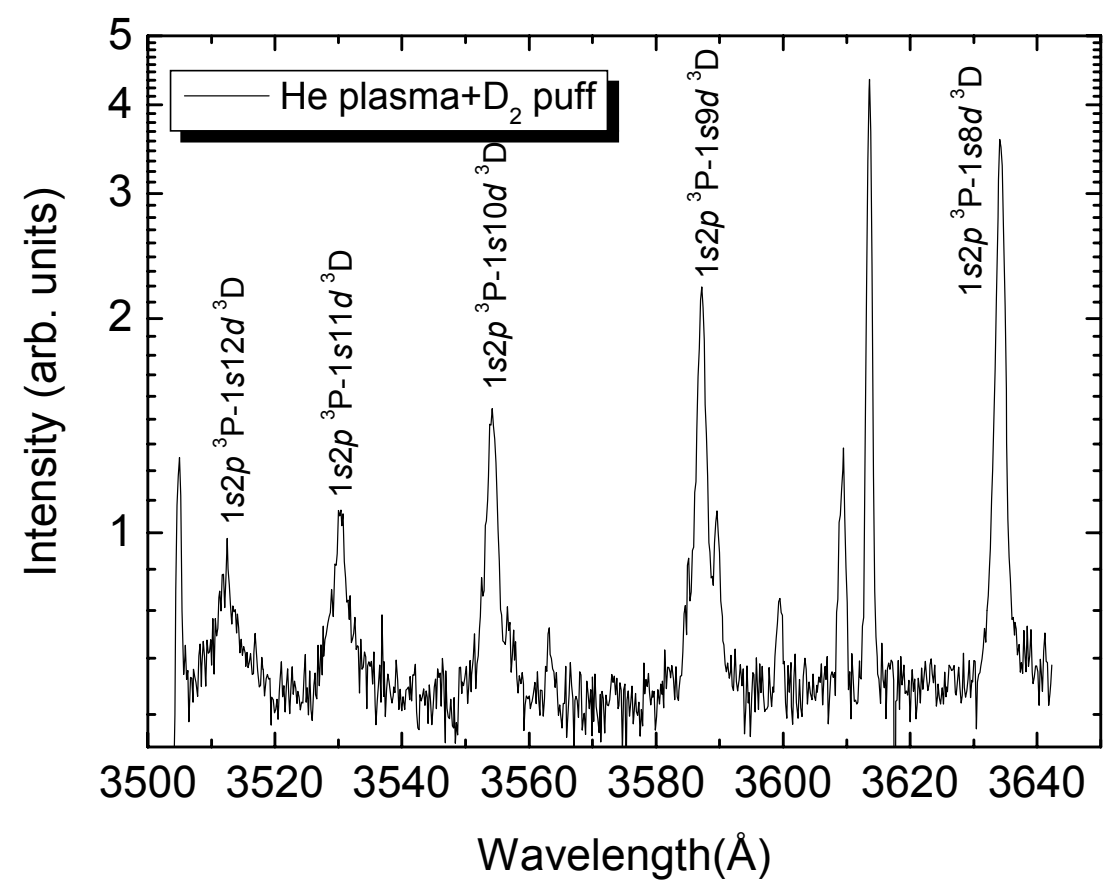

FIGURE 3. Spectral lines of He I $1 \mathrm{~s} 2 \mathrm{p}^{3} \mathrm{P}-1$ snd ${ }^{3} \mathrm{D}$ diffuse series emitted in the divertor of the JET tokamak.

Lines with upper principal quantum number $n=8$ to $n=12$ are seen on Fig. 3 , and are found to be dominated by Stark effect for typical divertor conditions $\left(\mathrm{N}_{\mathrm{e}}=5 \times 10^{12}\right.$ $\left.5 \times 10^{14} \mathrm{~cm}^{-3}, \mathrm{~T}_{\mathrm{e}}=0.2-5 \mathrm{eV}\right)$. For the $1 \mathrm{~s} 2 \mathrm{p}{ }^{3} \mathrm{P}-1 \mathrm{~s} 8 \mathrm{~d}{ }^{3} \mathrm{D}$ line at $3635 \AA$, estimates of the collision time $\tau \sim \mathrm{d} / \mathrm{v}_{\text {th }}$ (with $\mathrm{d}$ the average distance between charged particles, and $\mathrm{v}_{\text {th }}$ their thermal velocity) for ions and electrons, compared to the line time of interest $t_{1}$ (inverse of the line width), suggest that a standard Stark approach may be used, with the electrons described by an impact operator $\left(\tau_{\mathrm{e}}<<\mathrm{t}_{1}\right)$, and the ions by a static approximation $\left(\tau_{\mathrm{i}}>>\mathrm{t}_{1}\right)$. Using theoretical Stark line profiles, it will thus be possible to perform a density diagnostic by fitting the shape of the line. The line shape 
resulting from our model is plotted on Fig. 4 for a density of $\mathrm{N}_{\mathrm{e}}=5 \times 10^{13} \mathrm{~cm}^{-3}$, and for three temperatures $\mathrm{T}_{\mathrm{e}}=1,2$ and $5.8 \times 10^{4} \mathrm{~K}$. As the temperature is increased, thus reducing the impact width of the Stark components, it can be seen that the line exhibits satellite components near to the main line. The component appearing on both sides of the main peak of the line correspond to the forbidden transitions $2{ }^{3} \mathrm{P}-8^{3} \mathrm{P}$, ${ }^{3} \mathrm{~F},{ }^{3} \mathrm{G}$ which have been made observable by the mixing of the levels induced by the electric microfield.

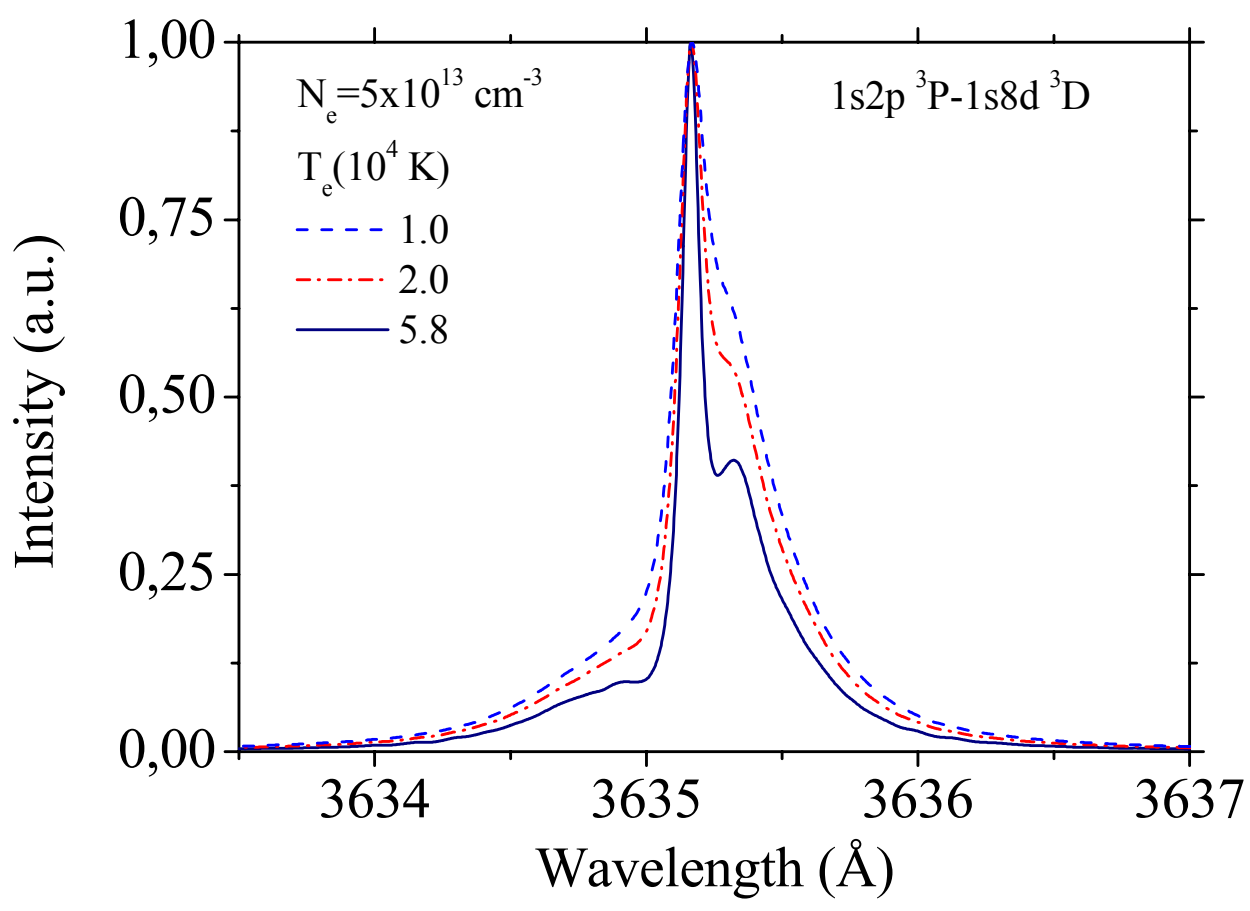

FIGURE 4. Model for the spectral line of He I $1 \mathrm{~s} 2 \mathrm{p}^{3} \mathrm{P}-1 \mathrm{~s} 8 \mathrm{~d}^{3} \mathrm{D}$, for $\mathrm{N}_{\mathrm{e}}=5 \times 10^{13} \mathrm{~cm}^{-3}$, and $\mathrm{T}_{\mathrm{e}}=1,2$ and $5.8 \times 10^{4} \mathrm{~K}$.

Note that divertor plasmas can be strongly inhomogeneous, and that in such cases a comparison to experimental spectra will require a specific analysis.

\section{Tokamaks with Dense Plasmas}

A few magnetic confinement fusion devices are already operated with densities of the order of $10^{14} \mathrm{~cm}^{-3}$ or higher. For such conditions, found for instance in the Alcator CMod tokamak, Stark broadening of lines emitted in the divertor plasma not only affects the high $\mathrm{n}$ lines, but has to be taken into account even for the low lying lines. The study of such conditions has recently gained a new interest with the decision of building the ITER tokamak, which is designed for high density steady state discharges. One should stress that some care must be taken for modeling the line 
shapes in such plasmas. First of all, the low lying lines will be affected simultaneously by Zeeman and Stark effects, requiring a specific approach $[12,13,14]$. Secondly, the standard Stark broadening approach will rarely be valid. Indeed, for low $\mathrm{n}$ lines, ion dynamics affects the line shapes, even for the highest densities found in divertors. On the other hand, the impact approximation for the electrons becomes more and more questionable as lines with large principal quantum numbers are considered $(n>10-12)$. Although it remains to perform a detailed analysis of the error made by using the binary impact approximation for cases where multiple electronic collisions act on the emitter, other models may be used to address this issue. Indeed, this problem is similar to the ion dynamics problem, since it involves many body dynamic interactions between charged particles and the emitter. Approaches such as simulations coupled to the integration of the Schrödinger equation, model microfield methods (MMM [15], FFM [16]), or analytical models [17] should be validated and compared before using them for extensive applications to divertor conditions.

\section{SPECTRAL ANALYSIS OF IMPURITIES}

Intrinsic and/or purposely seeded impurities are always present in magnetic confinement fusion (MCF) devices. Spectrometers observing plasma emission from the visible wavelength range up to the X-ray range are installed on these devices. Most spectroscopy is usually dedicated to the determination of both the plasma chemical composition (i. e., the identification of the impurity elements and the evaluation of their concentration) and the impurity transport coefficients. For this purpose only a few strong lines are considered and are simulated using impurity ion transport codes, that describe ionisation, recombination and radial transport of the ions of a given impurity species. They require as input data the radial profiles of the electron temperature $\mathrm{T}_{\mathrm{e}}(\mathrm{r})$ and of the electron density $\mathrm{N}_{\mathrm{e}}(\mathrm{r})$, which are needed to evaluate ionisation and recombination rate coefficients (respectively, $S_{\text {ion }}$ and $\alpha_{\text {rec }}$ ) and two transport coefficients, a diffusion coefficient $\mathrm{D}(\mathrm{r})$ and a convection velocity $\mathrm{V}(\mathrm{r})$.

Until recently, simulations of full spectra were less frequent. These include, e. g., full simulation in the XUV range of $n=2$ to $n=2$ L-shell Ne, Ar and Fe spectra [18, 19], of $\mathrm{n}=3$ to $\mathrm{n}=2 \mathrm{~L}$-shell Ne and Ar spectra [20,21], and of $\mathrm{n}=3$ to $n=3 \mathrm{M}$-shell Fe and Ni spectra $[22,23]$. The analysis of spectra emitted by hot and well-diagnosed magnetic fusion plasmas is important since it touches on atomic physics issues to be used in the analysis of high spectral resolution astrophysical observations, i.e. on the determination of the electron temperature, electron density and elemental abundances. In most cases atomic data are supposed to be valid and the simulations give $\mathrm{D}(\mathrm{r})$ and $\mathrm{V}(\mathrm{r})$, like, e.g., in Refs [20, 21]. But with high quality and large amount of spectroscopic data, it is possible to go further and to verify also $S_{\text {ion }}$ and $\alpha_{\text {rec. To the }}$ best of our knowledge, the first of such comparisons has been reported by May et al. [24], analysing in the FTU tokamak space-resolved distributions of laser blow-off (LBO) injections of $\mathrm{Fe}$ and $\mathrm{Ge}$ ions and of intrinsic Ni ions. Using data from Ref. [25] for $\mathrm{Fe}$ and $\mathrm{Ni}$ and from Ref. [26] for Ge, the F-like to B-like charge states could not be simulated unless the relevant $\alpha_{\text {rec }} / S_{\text {ion }}$ ratios were increased. In another paper on 
M-shell Ni spectra in the 144-180 A region [23] both D(r) and V(r) were known from similar discharges and with best fits on the strongest lines of the involved Ni X-XV ions, the best sets of data for both ionisation and recombination have been obtained.
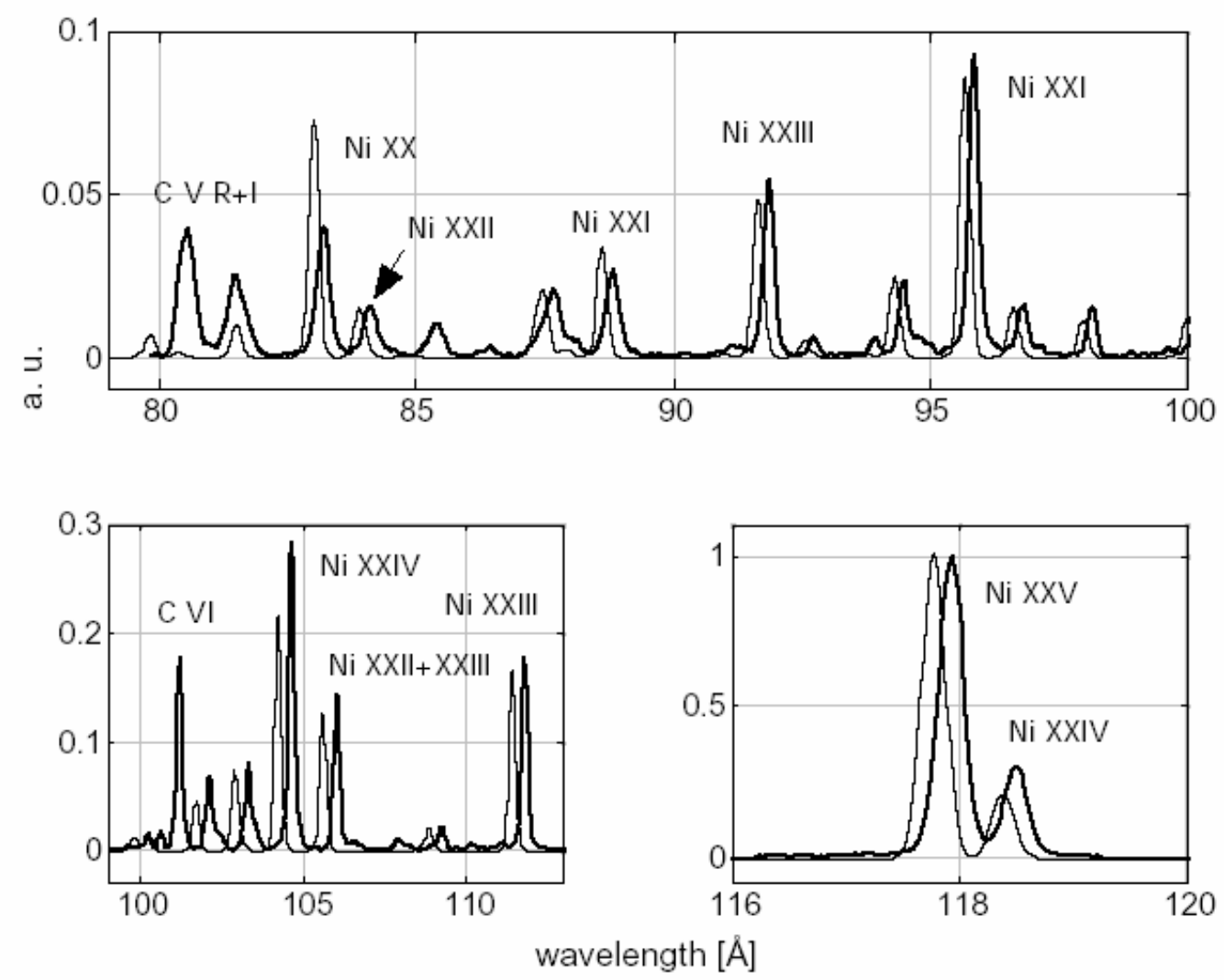

FIGURE 5. Experimental (thick line) and simulated (thin line) normalised Ni spectra from a limiter JET discharge heated by a 6 MW ICRH pulse with the most intense Ni and C line identified. For clearness, three partial spectral ranges are considered and the thin spectrum has been slightly shifted towards the blue.

The ionisation and recombination rates have to be regularly updated following the publication of new theoretical calculations and new experimental data. Astrophysicists usually consider only elements up to $\mathrm{Cu}(Z=29)$ or $\mathrm{Zn}(\mathrm{Z}=30)$. An article that updates recommended data from recent references is published every five or six years allowing determination of charge state distributions (CSD). At present, the most recent article in this context is the 1998 paper from Mazzotta et al. [25], which has been extended in Ref. [26] from $\mathrm{Cu}$ to $\mathrm{Ge}(\mathrm{Z}=32)$. Assessments and updates of these atomic data have been started both by us and at the Columbia University under the leadership of D. Savin [27]. Concerning both radiative (RR) and dielectronic (DR) recombinations it was concluded that, for highly ionised ions down to the recombining Na-like isosequence, the calculations from the $\mathrm{DR}+\mathrm{RR}$ coordinated program under the leadership of $\mathrm{N}$. Badnell of the University of Strathclyde should be used $[28,29]$ as reference calculations. Where measurements exist, these theoretical data agree with recent laboratory work to within typically $35 \%$ 
or better at the temperatures relevant for collisionally ionized plasmas. For the less ionized sequences (up to recombining Mg-like ions), the Columbia University group claims [27] that there are no new reliable recombination data, and that the Mazzotta's

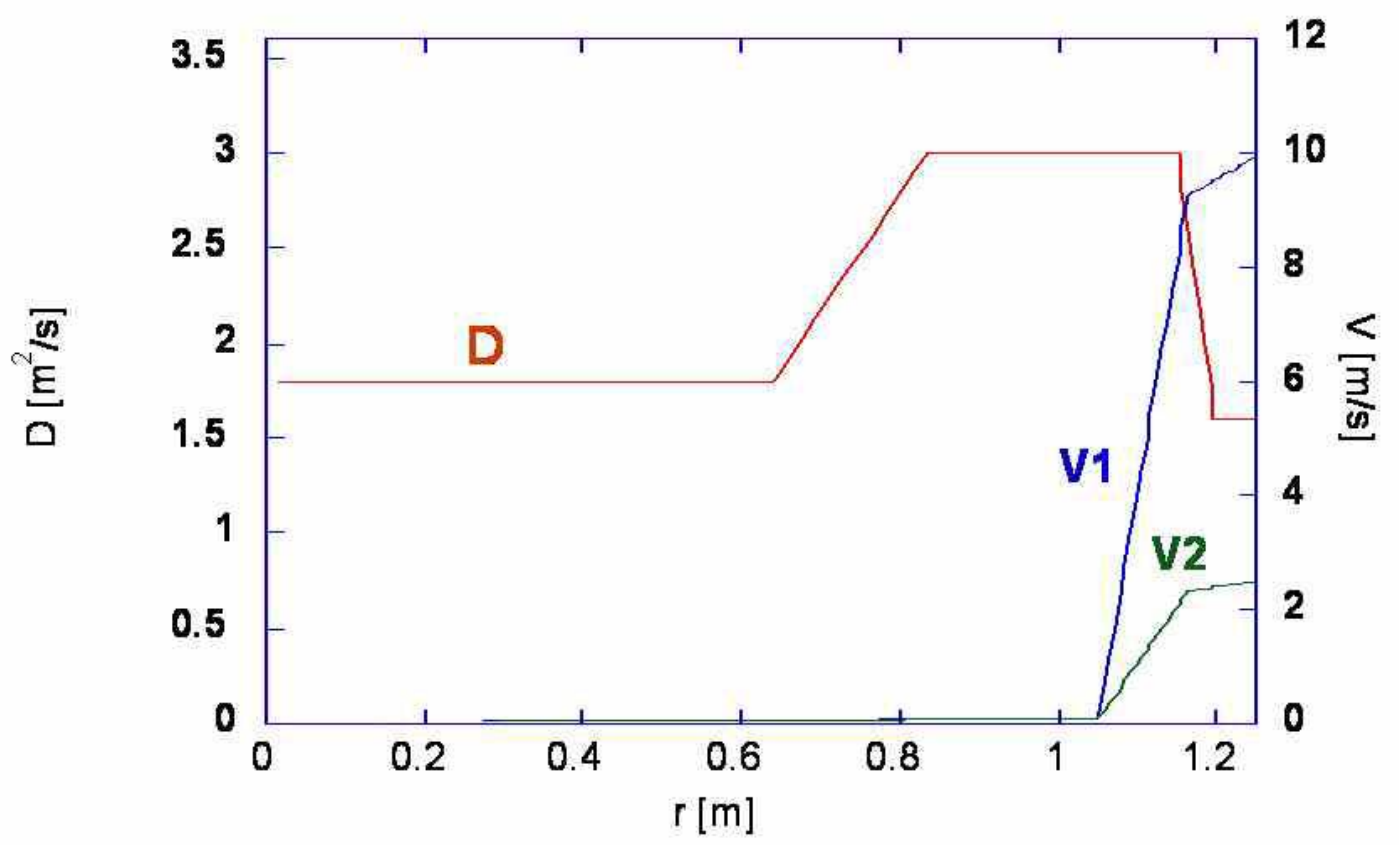

FIGURE 6. $\mathrm{D}(\mathrm{r})$ and $\mathrm{V}(\mathrm{r})$ radial profiles for the Mo-Ge LBO. To simulate closely the outflow of as many as possible ionisation stages, the simulation requires a time-dependent V(r). Critical for it is the simulation of the soft X-ray brightness. Shown are the limit profiles: V1 up to $100 \mathrm{~ms}$ and V2 between 300 and $600 \mathrm{~ms}$ after the injection. Inward velocities are positive.

rates should be kept for calculations of charge state distribution (CDS), in spite of the almost general use of the Burgess-Merts formula, which is known to underestimate the $\mathrm{DR}$ rates at low $\mathrm{T}_{\mathrm{e}}$.

A corresponding review of the available ionization data is not yet available and, at the present, Mazzotta's ionization rates should be kept as the reference ones to be used in CSD evaluations, the only exception being $\mathrm{Ni}$, since the assessed data of Ref. [30] were not considered. For $\mathrm{Kr}$ and Mo (elements without astrophysical interest) our review is presented in Ref. [31].

Here we present the analysis of two JET tokamak Ni and Mo spectra from predivertor limiter discharges. The intrinsic Ni spectrum in the 80-120 $\AA$ spectral range was obtained during a steady state phase of a $5 \mathrm{MA}$ discharge heated by $6 \mathrm{MW}$ ion cyclotron resonance heating $(\mathrm{ICRH})$ pulse $\left(\mathrm{T}_{\mathrm{e}}(0) \sim 5 \mathrm{keV}, \mathrm{N}_{\mathrm{e}}(0) \sim 210^{13} \mathrm{~cm}^{-3}\right)$. The Mo spectrum in the 35-50 $\AA$ spectral range was obtained with a LBO injection from a composite Mo-Ge target into a 3 MA discharge heated by a 8 MW ICRH pulse $\left(\mathrm{T}_{\mathrm{e}}(0) \sim 6 \mathrm{keV}, \mathrm{N}_{\mathrm{e}}(0) \sim 3.510^{13} \mathrm{~cm}^{-3}\right)$.

After evaluation of radial CSD, post-process subroutines are used to produce simulated spectra, using line photon emission coefficients (PECs) from collisionalradiative models (CRM). Like for Refs [18-23], the PECs have been obtained using the HULLAC code. 
We perform best fits of the simulated spectra to the peaks of the strongest lines (i.e., to the main contributors to the detected spectra). If $\operatorname{sp}_{\exp }(\lambda)$ and $\operatorname{sp}_{\text {sim }}(\lambda)$ are, respectively, the normalized experimental and simulated spectra, $\mathrm{C}_{\text {mult }}$ is a multiplicative constant, whose value is obtained by minimization of $\Sigma_{\mathrm{i}}\left(\mathrm{C}_{\text {mult }} \operatorname{sp}_{\text {sim }}\left(\lambda_{\mathrm{i}}\right)-\operatorname{sp}_{\exp }\left(\lambda_{\mathrm{i}}\right)\right)^{2}$ over the selected lines. If a verification of the atomic data is possible, the 'best' spectra are the ones with the lowest estimated sample deviation defined as the square root of the sum to be minimized.

Comparisons of experimental and simulated $\mathrm{Ni}$ spectra are given in Fig. 5, where, for clearness, the simulated thin line spectrum has been slightly shifted towards the blue. For this simulation both transport coefficients are the same as for the Ar seeded discharge of Ref. [21], implying, as found in several JET spectroscopic data analysis, the presence of a peripheral transport barrier, obtained reducing D and/or increasing $\mathrm{V}$ inward. The lack of other experimental spectroscopic data and/or of time resolved spectra hinders any verification of the used atomic data. Intensity ratios of L-shell lines can be density-sensitive and applicable to the diagnostic of astrophysical sources. In Ref. [19] experimental Fe line ratios are presented and compared with the predictions of the HULLAC code. Corresponding not-discussed Ni line ratios of the spectrum of Fig. 5 appear to be in reasonable agreement with the predictions.
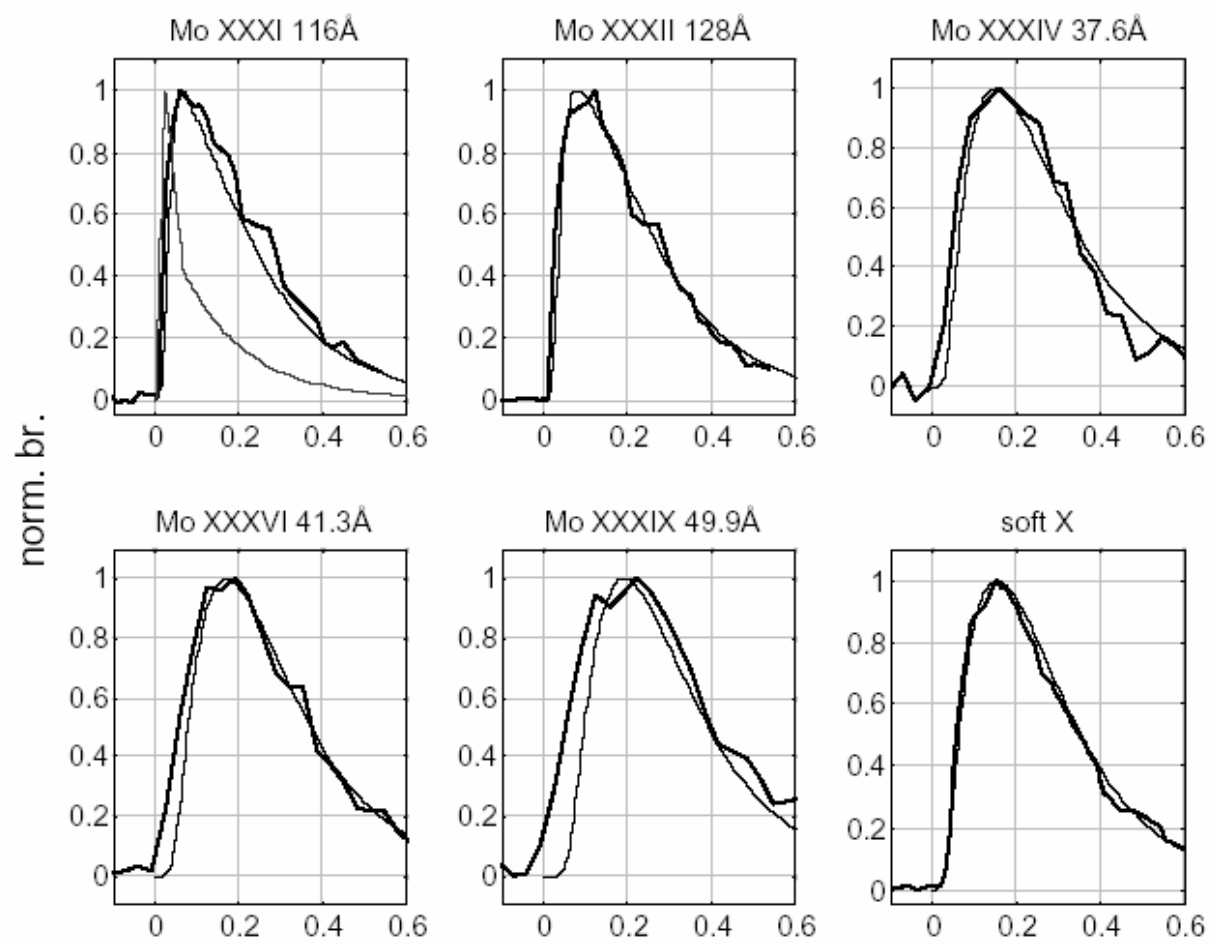

$\mathrm{t}[\mathrm{s}]$

FIGURE 7. Experimental (thick line) and simulated (thin line) normalised brightnesses. The additional thin line (superposed to the Mo XXXI line) shows the Mo XIII line brightness, following the incoming neutral flux. 
The transport coefficients $\mathrm{D}(\mathrm{r})$ and $\mathrm{V}(\mathrm{r})$ of the Mo-Ge LBO (shown in Fig. 6) have been obtained by simulations of Mo and Ge line brightnesses. They show the presence of both a peripheral barrier at the plasma edge and of an improved confinement core region.

As a general remark, it should be stressed that the values found for D by this fit are much larger than those predicted by theories based on collisional diffusion (so called neoclassical diffusion). The reason for the larger observed values is that the diffusion is dominated by turbulence both for impurities and the whole plasma. The question of turbulence and its possible alteration of line shapes will be introduced in the last section.

In Fig. 7 six normalized brightnesses are shown. The boundary condition (source function) of the incoming neutral flux at the last mesh has been chosen to follow the lowest ionisation stages observed [31] (Mo XXIV-XXV and Ge XXI).

The relative amplitudes of the lines from different ionisation stages depend on the transport parameters and on the atomic rates. The 35-50 $\AA$ experimental spectrum, obtained $200 \mathrm{~ms}$ after the LBO injection, is shown (solid) in Fig. 8. The not-shown simulated spectrum shows B-like and Be-like ion lines clearly stronger than found experimentally. It is then necessary to modify either the recombination or the ionisation rates of the involved iso-sequences to reduce the core ionisation degree. One of the satisfactory simulations is shown (thin line, slightly shifted towards the blue for clearness) in Fig. 8. It has been obtained by multiplying, for recombining Flike to Be-ions, the $\alpha_{\text {rec }}$ rates by the following multiplicative factors $\mathrm{f}=1.0,1.1,1.6$, $1.8,1.25,1.25$. The simulation was practically equivalent (i.e., with practically the same estimated sample deviation) by dividing by $\mathrm{f}$ the $\mathrm{S}_{\text {ion }}$ rates for ionising Ne-like to B-like ions. An idea of the uncertainties associated with the choices of the multiplicative factors $f$ is given by the fact that the sample deviations are similar when decreasing and increasing the f-values by about $0.1-0.15$ for two consecutive ionisation stages.

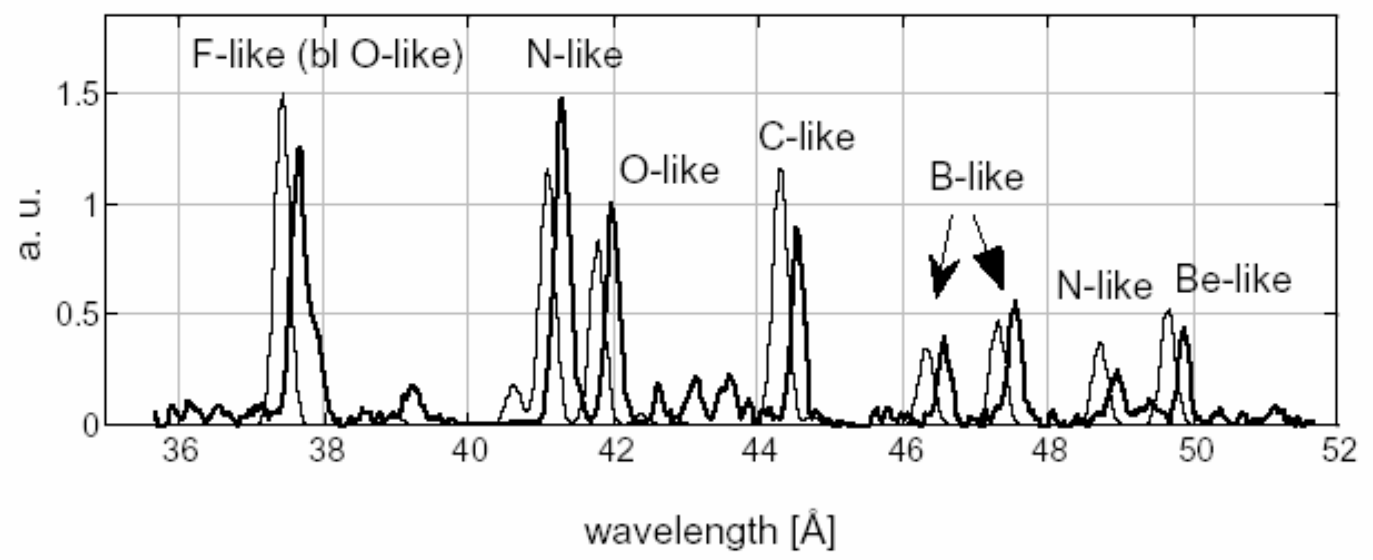

FIGURE 8. Experimental (thick line) and simulated (thin line) spectra, $200 \mathrm{~ms}$ after the LBO injection. For clearness, the thin spectrum has been slightly shifted towards the blue. The recombination rates of the six shown iso-sequences have been increased as explained in the text. 
It is known that, both in astrophysics and in MCF devices, the CSD is reduced by recombining charge exchange $(\mathrm{CX})$ collisions. In the latter devices, the considered $\mathrm{CX}$ collisions are with neutral hydrogen isotopes with poorly known radial profiles. Moreover, excepted for light elements, only scaled cross sections versus scaled energies are available.

The CRMs generally consider each ion independently from others ignoring ionisation and recombination into and out of the ground and excited energy levels. Processes included are radiative decays and electron collisions (proton collisions are sometimes included only between the lowly lying states).

In the Marseilles laboratory, CRMs have been considered adding CX reactions populating the excited states [32]. In fact, a self-consistent analysis of the particle transport and charge exchange (including also charge exchange from excited states) with a self-consistent multilevel collisional-radiative approach has shown that charge exchange phenomena, particle transport and atomic data are very closely related, as proved for the argon spectra in Ref. [33].

\section{EFFECT OF HYDRODYNAMIC TURBULENCE}

Edge plasmas of tokamaks are affected by low frequency turbulence. This turbulence is produced by small scale instabilities, induced by density, temperature and velocity gradients. Here we focus on drift wave turbulence, whose effects on the fluctuation of hydrodynamic variables has been studied by fluid models [34]. Such models predict fluctuations rates for the velocity, density and temperature reaching several tens of percent. For tokamak edge plasmas their characteristic fluctuation time is of the order of a few $10 \mu \mathrm{s}$, and their typical spatial length is about 10 Larmor radius $(\sim \mathrm{cm})$. This characteristic turbulent time is usually much larger than the typical time scale of the atomic processes, such as for instance the line shape time of interest. On the other hand, the acquisition time $\tau_{\mathrm{m}}$ for a line shape measure is generally much larger than this turbulent fluctuation time. A line shape measurement thus performs a spatial and time average over fluid cells having each a specific set of values for the density, velocity and temperature

$$
\mathrm{I}_{\mathrm{mes}}(\omega)=\frac{1}{\tau_{\mathrm{m}}} \int_{0}^{\tau_{\mathrm{m}}} \frac{1}{\mathrm{~L}} \int_{0}^{\mathrm{L}} \mathrm{I}_{0}(\omega, \mathrm{z}, \mathrm{t}) \mathrm{dzdt},
$$

where $\mathrm{I}_{0}$ is the local profile at location $\mathrm{z}$ and time $\mathrm{t}$, and $\mathrm{L}$ is the length of the line of sight. It has been shown [5] that the measured profile may be expressed as an integral over the fluid variables of the local profile, weighed by the probability density function (PDF) of the fluctuating hydrodynamic variables. The PDF of only one hydrodynamic variable has been studied theoretically $[35,36]$, and has been measured in a few devices [37,38]. Since this quantity is of great interest for characterizing the edge plasma turbulence, the link made with the line shape may lead to a valuable diagnostic of turbulence by spectroscopy. Recent advances in this direction for Doppler line shapes [7] and Stark profiles [39] are reported in this volume. 


\section{CONCLUSION}

Spectral lines have been extensively used since the beginning of spectroscopic diagnostics in magnetic fusion. The observation of line intensities, widths and shifts brings information on the plasma parameters and dynamics. A reliable diagnostic requires the use of realistic atomic data, atomic kinetics, and line shape models. The complex recycling process of hydrogen isotopes is clarified by the observation of neutral deuterium Balmer alpha lines broadened by Doppler and Zeeman effects. An accurate model for this diagnostic requires the full calculation of Zeeman effect by retaining the fine structure. A similar model is necessary for the calculation of opaque Lyman transitions used in Monte Carlo codes for predicting the divertor conditions. Stark effect affects line shapes of hydrogen and helium lines emitted from levels of high principal quantum numbers. These lines are used for an electronic density diagnostic in the edge regions. For the condition of ITER, Stark effect will affect most of the hydrogen lines due to higher charged particles densities. Models designed to perform a density diagnostic will thus often require the treatment of many body dynamic effects. As a general remark, an improved treatment of the spectral line shape should consider simultaneously atomic kinetics, radiation transport and diffusion processes that would affect the observations performed from non-uniform, non-equilibrium and non-stationary tokamak plasmas with complex composition.

Spectroscopy in the core plasma concerns principally the impurities. The fit of their spectra simultaneously improves the knowledge of atomic data, and the complex physics of transport. Remarkably, such fits indicate that the impurity transport is turbulent, this phenomena being especially marked while approaching the edge region. Since it is the whole plasma which is turbulent, this has been the starting point for an analysis of a possible role of hydrodynamic turbulence on the line shapes.

\section{ACKNOWLEDGMENTS}

This work is part of a collaboration (LRC DSM 99-14) between the Laboratory of Physique des Interactions Ioniques et Moléculaires and the association Euratom-CEA in Cadarache. The work of K.B. Fournier has been performed under the auspices of the US Department of Energy by the University of California Lawrence Livermore National Laboratory under Contract No. W-7405-Eng-48.

\section{REFERENCES}

1. S. Suckewer and E. Hinnov, Phys. Rev. Lett. 41, 756 (1978)

2. J. D. Hey, C.C. Chu, P. Mertens, S. Brezinsek, $17^{\text {th }}$ Int. Conf. Spectral Line Shapes,

Ed. E. Dalimier, Frontier group 2004, p.207

3. B. Welch et al., $13^{\text {th }}$ Int. Conf. Spectral Line Shapes, Ed. M. Zoppi, AIP 386, p.113

4. R. C. Isler et al., Phys. Plasmas $\underline{6}, 541$ (1999)

5. Y. Marandet et al., Europhysics Letters 69 , 531(2005) 
6. Ph. Mertens et al., Plasma Phys. Control. Fusion $\underline{43}$, A349 (2001)

7. Y. Marandet et al., this volume

8. D. Reiter, S. Wiesen and M. Born, Plasma Phys. Control. Fusion 44, 1723 (2002)

9. V. Kotov et al., to appear in Contributions to Plasma Physics

10. H. R. Griem, A. C. Kolb and K. Y. Shen, Phys. Rev. 116, 4 (1959)

11. J. Rosato et al., to appear in Journal of Nuclear Materials.

12. S. Günter and A. Könies, JQSRT 62, 425 (1999)

13. L. Godbert-Mouret et al., JQSRT 71, 365 (2001)

14. M. Adams et al., Phys. Rev. E $\underline{66}, 066413$ (2002)

15. C. Stehlé, Eur. Phys. J. D. 11, 491 (2000)

16. B. Talin et al., Phys. Rev. A $\underline{51}, 4917$ (1995)

17. D. B. Boercker, C. A. Iglesias, and J. W. Dufty, Phys. Rev. A $\underline{36}, 2254$ (1987)

18. M. Mattioli M et al., Plasma Phys. Control. Fusion $\underline{44}, 33$ (2002)

19. K. Fournier et al., Astrophys. J. 561, 1144 (2001)

20. M. Mattioli et al., Phys. Rev. E $\underline{60}, 4760$ (2000)

21. M. Mattioli et al., J. Phys. B $\underline{34}, 127$ (2001)

22. K. Fournier et al., Astrophys. J. 550, L117 (2001)

23. M. Mattioli et al., J. Phys. B 37, 13 (2004)

24. M. May et al., Phys. Rev. E $\underline{64}, 036406$ (2001)

25. P. Mazzotta et al., Astron. \& Astrophys. Suppl. 133, 403 (1998)

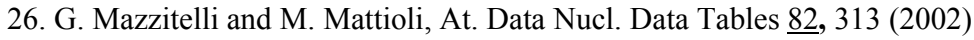

27. P. Bryans et al., to be published in Astrophys. J. Suppl.

28. N. Badnell N et al., Astron.\& Astrophys. 406, 1151 (2003) and following articles in A\&A

29. N. Badnell, submitted to Astrophys. J. Suppl.

30. M. Pindzola et al., Physica Scripta T37, 35 (1991)

31. M. Mattioli et al., to appear in J. Phys. B

32. F.B. Rosmej, R. Stamm, V. Lisitsa, Europhysics Letters 73 , 342 (2006)

33. F.B. Rosmej et al., Plasma Phys. and Control. Fusion $\underline{41}, 191$ (1999)

34. W. Horton, Rev. Mod. Phys. 71, 735 (1999)

35. Y. Marandet and J. Dufty, Contrib. Plasma Phys., $\underline{46}$ (7-9), 197 (2006)

36. J. Krommes, Phys. Reports $\underline{360}, 1$ (2002)

37. J. Graves et al., Plasma Phys. Control. Fusion 47, L1(2005)

38. A. Ferreira, Plasma Phys. Control. Fusion 46, 669(2004)

39. J. Rosato et al., this volume 